\title{
Structural insights into a novel histone demethylase PHF8
}

Lin $\mathrm{Yu}^{1,{ }^{*}}$, Yang Wang ${ }^{1, *}$, Shuo Huang ${ }^{2,{ }^{*}}$, Jianjun Wang ${ }^{1}$, Zengqin Deng ${ }^{1}$, Qi Zhang ${ }^{1}$, Wei Wu ${ }^{1}$, Xingliang Zhang ${ }^{1}$, Zhao Liu ${ }^{1}$, Weimin Gong ${ }^{3}$, Zhongzhou Chen ${ }^{1}$

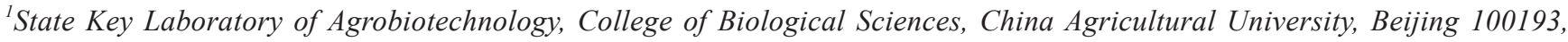
China; ${ }^{2}$ Department of Biochemistry and Molecular Biology, National Key Laboratory of Medical Molecular Biology, Institute of Basic Medical Sciences, Chinese Academy of Medical Sciences and Peking Union Medical College, Beijing 100005, China; ${ }^{3}$ State Key Laboratory of Biomacromolecules, Institute of Biophysics, Chinese Academy of Sciences, 15 Datun Road, Beijing 100101, China

Dynamic regulation of histone methylation/demethylation plays an important role during development. Mutations and truncations in human plant homeodomain (PHD) finger protein 8 (PHF8) are associated with X-linked mental retardation and facial anomalies, such as a long face, broad nasal tip, cleft lip/cleft palate and large hands, yet its molecular function and structural basis remain unclear. Here, we report the crystal structures of the catalytic core of PHF8 with or without $\alpha$-ketoglutarate $(\alpha-\mathrm{KG})$ at high resolution. Biochemical and structural studies reveal that PHF8 is a novel histone demethylase specific for di- and mono-methylated histone H3 lysine 9 (H3K9me2/1), but not for $\mathrm{H3K9me3.} \mathrm{Our} \mathrm{analyses} \mathrm{also} \mathrm{reveal} \mathrm{how} \mathrm{human} \mathrm{PHF8} \mathrm{discriminates} \mathrm{between} \mathrm{methylation} \mathrm{states} \mathrm{and} \mathrm{achieves}$ sequence specificity for methylated H3K9. The in vitro demethylation assay also showed that the F279S mutant observed in clinical patients possesses no demethylation activity, suggesting that loss of enzymatic activity is crucial for pathogenesis of PHF8 patients. Taken together, these results will shed light on the molecular mechanism underlying PHF8-associated developmental and neurological diseases.

Keywords: PHF8 (PHD finger protein 8), histone demethylase, chromatin modification, methylated H3K9, crystal structure, $\mathrm{X}$-linked mental retardation (XLMR), facial anomalies

Cell Research (2010) 20:166-173. doi: 10.1038/cr.2010.8; published online 26 January 2010

Histone methylation plays important roles in multiple biological processes including transcription, signal transduction, development, cellular proliferation and differentiation $[1,2]$. For example, lysine methylation at H3K9 is usually associated with regions of transcriptionally silenced chromatin. Recent studies indicate that histone methylation can be removed by two distinct classes of histone demethylases, namely, flavin adenine dinucleotide (FAD)-dependent [3] and JMJC-containing protein families [4-7]. The reversible process of histone methylation and demethylation has been implicated in multiple biological processes including heterochromatin formation, $\mathrm{X}$-inactivation, genomic imprinting and silencing of

\footnotetext{
*These three authors contributed equally to this work. Correspondence: Zhongzhou Chen

Tel: +86-10-62734078

E-mail: chenzhongzhou@cau.edu.cn

Received 12 October 2009; revised 22 November 2009; accepted 15 December 2009; published online 26 January 2010
}

homeotic genes. Aberrant histone methylation has been linked to a number of human diseases such as leukemia and prostate cancers [8].

Human plant homeodomain (PHD) finger protein 8 (PHF8) contains a PHD-type zinc-finger domain, a JMJC domain, a vacuolar domain and a coiled coil stretch $[2$, 9, 10]. In vivo, the PHF8 transcript shows a ubiquitous expression pattern [11]. It is highly and widely expressed in many tissues, such as testis, lung and tumor tissues. It was found that defects in PHF8 were associated with diseases such as cleft lip, cleft palate, X-linked mental retardation (XLMR) and pathogenesis of autism [12]. These findings link PHF 8 to cognitive function and midline formation [13]. It was also proposed that PHF8 participates in regulating cellular growth, development and differentiation [14].

However, as no enzymatic activities, biochemical function or molecular structure of PHF 8 have been identified thus far, the function of PHF8 in development remains to be elucidated. Alignment studies and 
phylogenetic analysis [10] of the PHF2/PHF8 subfamily suggest that it is most closely related to the JHDM1 subfamily (known as FBXL11, KDM2 [15]). Both the catalytic process and the regulation of demethylation, however, are complicated. Furthermore, while the sequence similarity between the HIF (hypoxia-inducible factor) inhibiting factor FIH $[16,17]$ and JMJC histone demethylases is mostly found within the JMJC domain, additional diverse domains are essential for the activities of these enzymes. A high-resolution structure of PHF8 would provide invaluable information regarding the structure-function relationship of the PHF2/PHF8 subfamily. Here, we show that human PHF8 can demethylate $\mathrm{H} 3 \mathrm{~K} 9 \mathrm{me} 2 / 1$ and solve the crystal structures of the catalytic-core domain of PHF8 with and without $\alpha$-ketoglutarate $(\alpha-\mathrm{KG})$ in the presence of $\mathrm{Fe}^{2+}$.

\section{Results and Discussion}

PHF8 is a novel H3K9me2/me1-specific demethylase

To determine whether PHF8 is a histone demethylase, recombinant PHF8 was incubated with histones in the presence of $\mathrm{Fe}^{2+}$ and $\alpha-\mathrm{KG}$. The reaction was analyzed by western blotting [6]. The results (Figure 1A) show that PHF8 efficiently demethylates H3K9me2 and partially demethylates $\mathrm{H} 3 \mathrm{~K} 9 \mathrm{me} 1$, but not $\mathrm{H} 3 \mathrm{~K} 9 \mathrm{me} 3$. In addition, the levels of the other methylated histone peptides examined in this study remained unchanged (Supplementary information, Figure S1). To further confirm the demethylation, MALDI-TOF mass spectrometry was used to detect the change of the methylation state of histone peptides $[4,5,18]$. This protein exhibited demethylase activity toward the $\mathrm{H} 3 \mathrm{~K} 9 \mathrm{me} 2 / \mathrm{me} 1$ peptides, as
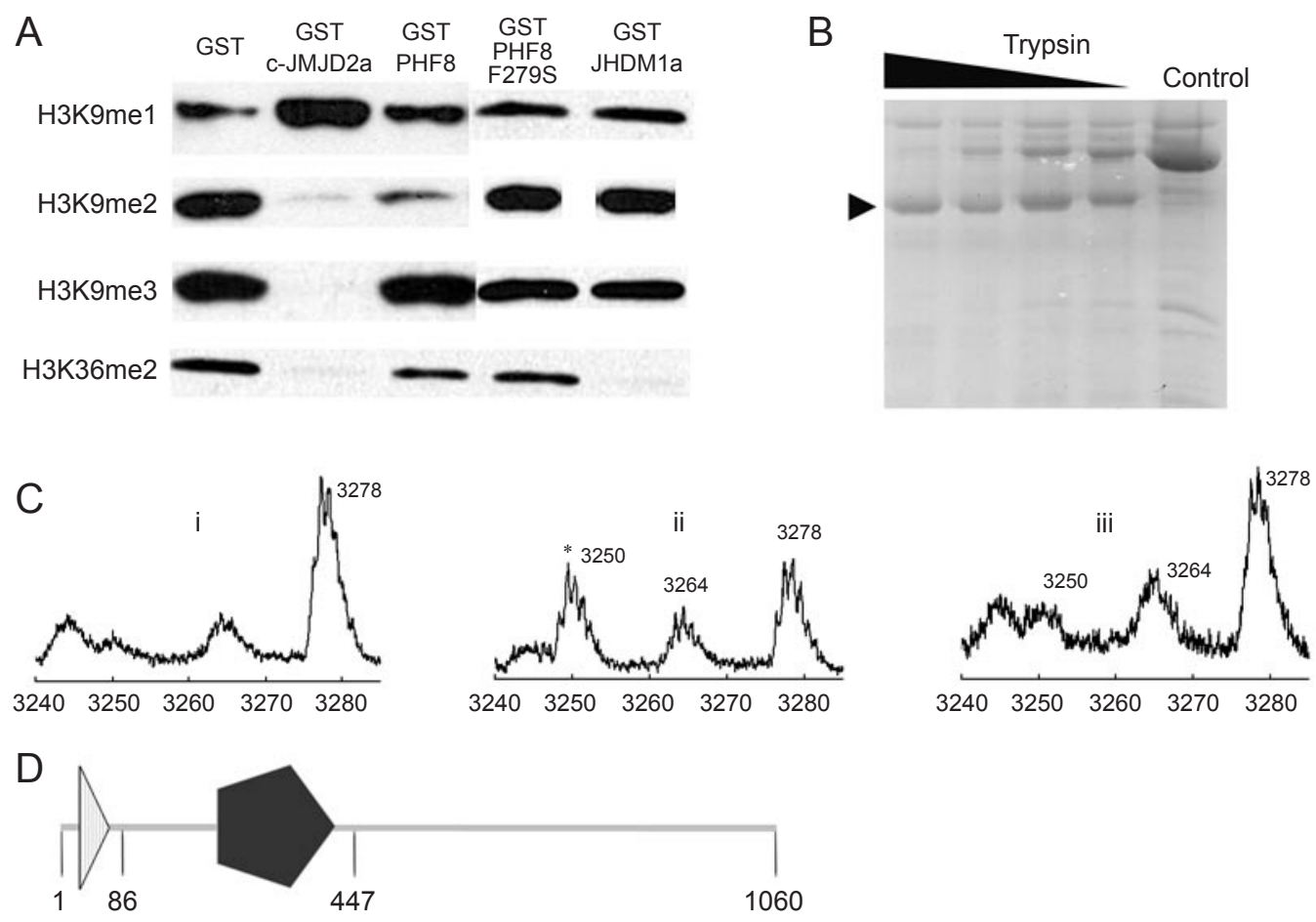

447

1060

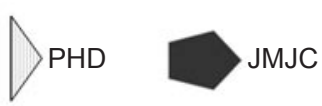

Figure 1 PHF8 is a histone H3K9Me2/1 demethylase. (A) GST-PHF8 demethylates H3K9me2 and H3K9me1. HeLa cell core histones were incubated in the presence of GST, GST-c-JMJD2a, GST-PHF8 (1-447), GST-PHF8 F279S (1-447) and GSTJHDM1a (1-517). Following the demethylation reaction, histone methylation levels were analyzed by western blotting with modification-specific antibodies. (B) A stable fragment of PHF8 is formed by limited proteolysis by trypsin. The control indicates the protein (residues 1-447) before digestion, and the stable fragment is labeled as a triangle and was confirmed as the C-terminal domain (residues 77-447) by protein sequencing and mass spectrometry. (C) Identification of histone demethylase activity for PHF8 by MALDI-TOF mass spectrometry. (i) Peptide control; (ii) GST-PHF8 1-447; and (iii) GST-PHF8 86-447. The appearance of a peak corresponding to unmethylated peptide is marked with a star. (D) Schematic of PHF8. The fragment of residues 86 to 447 was determined as the catalytic core of PHF8 (c-PHF8), and used for structural and functional characterization. 
A
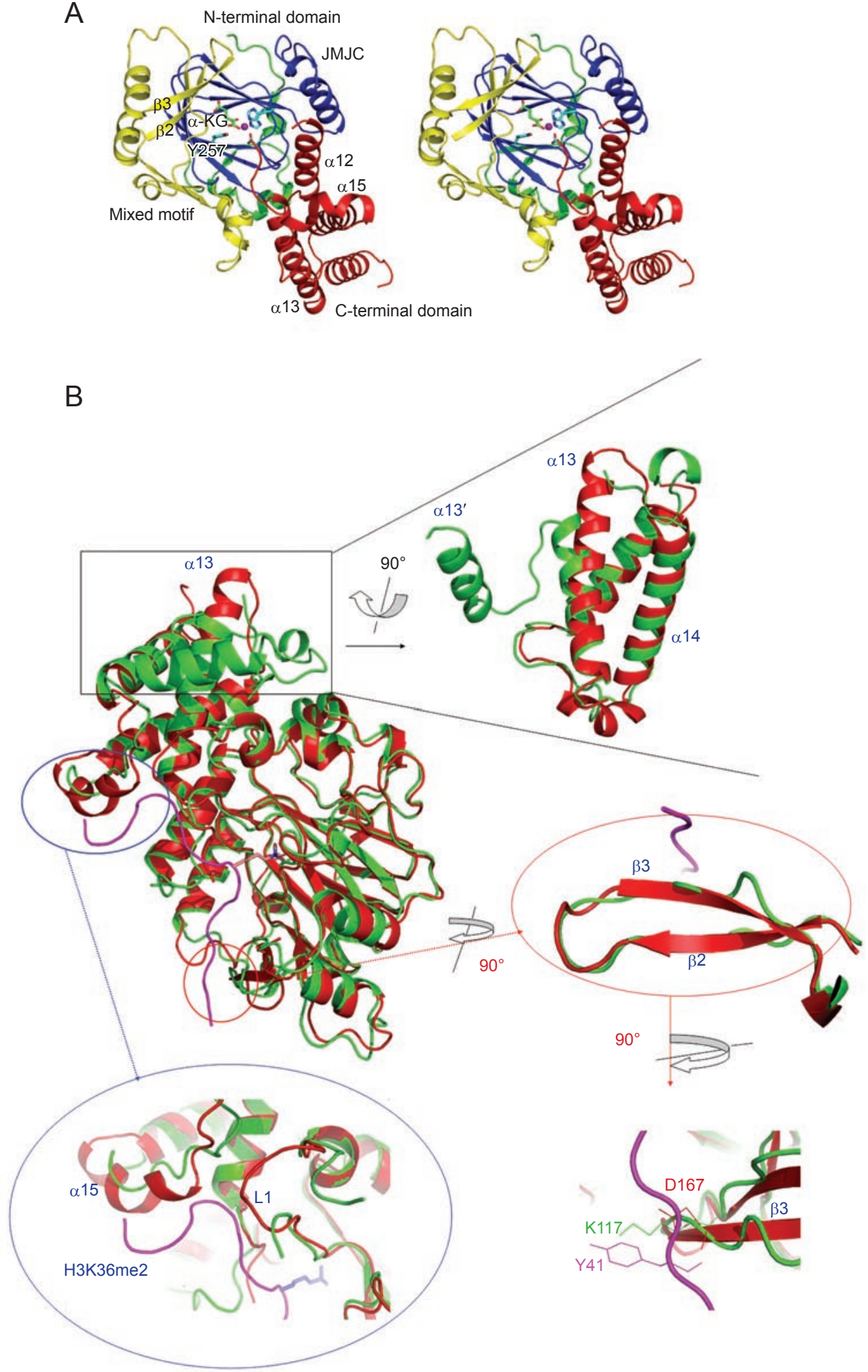

Figure 2 Structure of c-PHF8 and superposition with JHDM1a. (A) The overall structures of c-PHF8 in the presence of $\alpha-K G$ and $\mathrm{Fe}^{2+}$. The Fe ion is colored purple. Active center, $\alpha-K G$ and key secondary structures are labeled. (B) Structural superposition of c-PHF8 and JHDM1a (pdb: 2YU1) [23] by the Dali server. c-PHF8 and JHDM1a are colored red and green, respectively. The putative H3K36me2 (purple), extracted from the complex structures of c-JMJD2a (PDB:2P5B [24]) aligned with c-PHF8 by the Dali server [26], would be repulsed by c-PHF8, while it is suitably positioned for JHDM1a. Close-up views show the differences between c-PHF8 and JHDM1a in detail. 
indicated by the appearance of the peaks with mass of 3 264 and 3 250, which are $14 \mathrm{Da}$ and $28 \mathrm{Da}$ less than the original peptide (corresponding to loss of one and two methyl groups), respectively (Figure 1C). Unlike members of the JMJD2 subfamily (also known as JHDM3 and KDM4 [19, 20]), PHF8 can directly demethylate H3K9me2 to unmodified H3K9. These data verify that PHF8 is a novel histone H3K9me2/me1 demethylase.

To determine the 3D structure of PHF 8, we first unsuccessfully attempted to crystallize the full-length protein. Limited proteolysis with trypsin produced a stable fragment with a molecular weight of about 40 $\mathrm{kDa}$ (Figure 1B). N-terminal sequencing and mass spectrometry identified the fragment as residues 77-447 of PHF8, which lacks the preceding PHD domain. A recombinant PHF8 fragment encompassing residues 77447 also did not yield crystals. Removing an additional nine amino acids at the N-terminus (residues 86-447, herein called as c-PHF8, Figure 1D) produced welldiffracting crystals. Compared with the 1-447 fragment of PHF8, c-PHF8 has markedly lower demethylase activity (Figure 1C and Supplementary information, Figure S2), which suggests that the PHD domain and the flexible linker connecting the JMJC domain are important for enzymatic activity [21, 22], most likely by facilitating optimal binding of the substrate.

\section{Overall structure of c-PHF8}

The overall structure of c-PHF 8 consists of 16 $\alpha$-helices and $12 \beta$-strands (Figure 2A, Supplementary information, Figure S3), arranged in a manner similar to the structure of known JMJC domain-containing histone demethylases. Interestingly, a strong hydrophobic core, composed of F246, I248, F279, W282, F292, F293, I318, I349 and L353, is adjacent to the active center (Figure $3)$. We also tried to co-crystallize it with an $\mathrm{H} 3 \mathrm{~K} 9 \mathrm{me} 2$ peptide and $\alpha-K G$, but the former cannot be located in the electron density map. In the native c-PHF8, an iron ion is found in the active center. The iron ion is chelated by three absolutely conserved residues: His247, Glu249 and His319 (Figure 3, Supplementary information, Figure S3). The binding of $\alpha-K G$ does not induce significant conformational changes, and the structure has a rootmean-square deviation (RMSD) of $0.5 \AA$ compared to the native structure. In the complex structure, $\alpha-K G$ binds the iron ion at the active center mentioned above. In addition, T244 and K264 also stabilize $\alpha-K G$ through hydrogen bonds. Interestingly, the side chain of Y257 in the complex structure moves to the active center and binds to $\alpha-\mathrm{KG}$, indicating the important role of Y257 in stabilizing $\alpha-K G$ through a hydrogen bond. More importantly, the side chain of Y257 in the complex
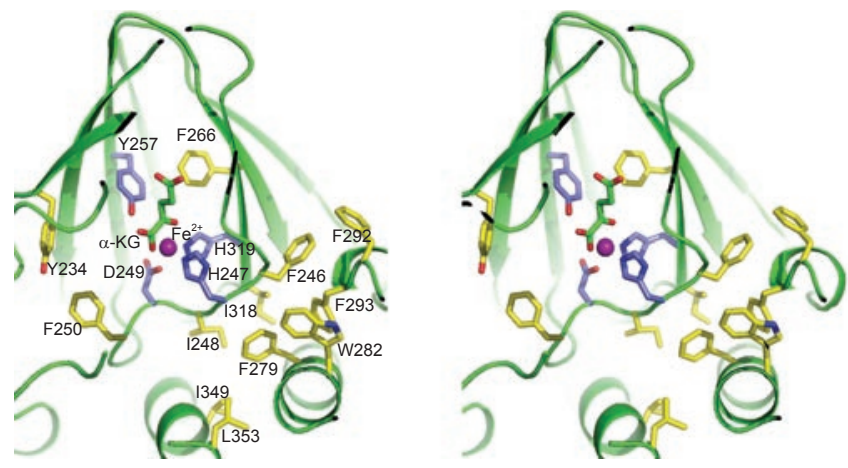

Figure 3 Catalytic center of c-PHF8 is stabilized by the hydrophobic core of F279, F292, F293, W282, F246, I248, I318, I349 and L353.

structure turns toward the substrate-binding channel (Figure 3 ) and is in a position to directly contact the methyl group of dimethylated $\mathrm{H} 3 \mathrm{~K} 9$.

Comparison of the structures of JMJC domain-containing histone demethylases

Superposition of the JMJC domains of PHF 8, hJHDM1a (PDB 2YU1 [23]) and JMJD2a (PDB 2P5B [24] and 2OX0 [25]) reveals conserved characteristics of $\alpha-\mathrm{KG} / \mathrm{Fe}^{2+}$-dependent histone demethylases. Compared to c-JMJD2a, no zinc-finger motif is found in c-PHF8. The overall RMSD between the C-alpha atoms of c-PHF 8 and that of c-JMJD2a is $3.8 \AA$, calculated by the Dali server [26]. Therefore, their structures differ significantly, despite having the same H3K9me2 substrate. The overall RMSD between PHF 8 and JHDM1a is $1.7 \AA$. Yet, interestingly, the substrates of these more closely related structures are totally different.

Most notable differences between the c-PHF 8 and JHDM1a structures are as follows: first, the folds of their C-terminal domains diversify greatly. The $\alpha 13$ of c-PHF 8 is approximately one-and-a-half turns longer than that of JHDM1a (Figure 2B and Supplementary information, Figure S3), which has a 99-residue insertion in $\alpha 13$. Most of this insertion region is disordered and the ordered portion, which includes a helix $\left(\alpha 13^{\prime}\right)$ and the connecting loop, stabilizes loop L1 between $\alpha 7$ and $\beta 5$ (residues W220 to K233 in PHF8, corresponding to residues W173 and Q197 in JHDM1a, Figure 2B) that may promote the binding of methyl $\mathrm{H} 3 \mathrm{~K} 36$ peptide to JHDM1a [23]. In addition, the helix $\alpha 13^{\prime}$ and the flanking loop also change the conformation of $\alpha 15$ to loop in JHDM1a and move a little away from H3K36 (Figure 2B and Supplementary information, Figure S3). Moreover, H3K36 cannot fit well with the surface of c-PHF8 (Figure 

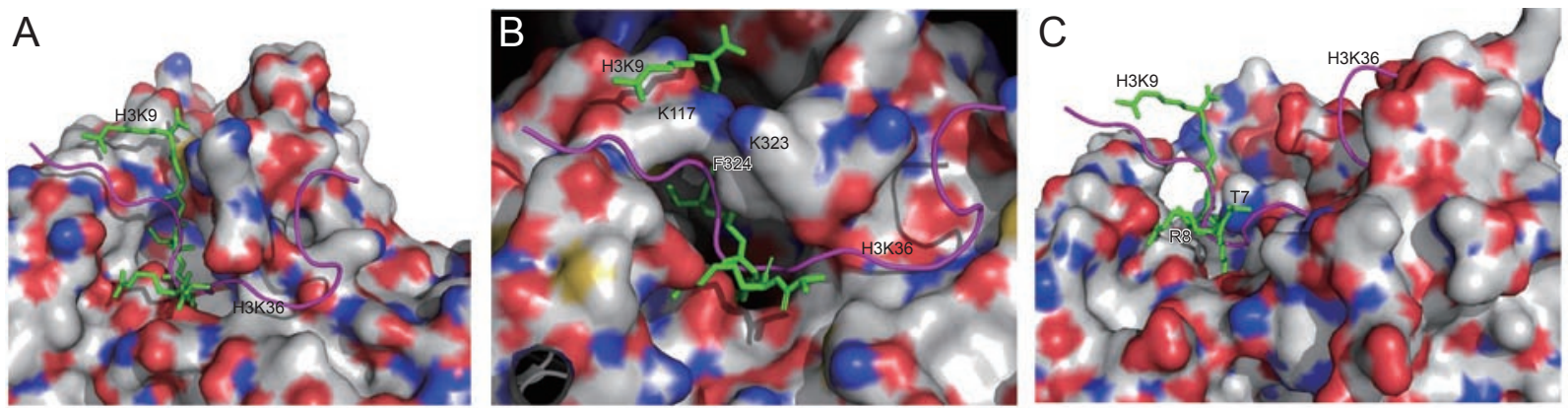

Figure 4 Model of H3K9 (green) and H3K36 (purple) peptides on the surface of c-JMJD2a (A), JHDM1a (B) and c-PHF8 (C). Methyl H3K9 and H3K36 in model were extracted from the complex structures of C-JMJD2a (PDB: 2OX0 [25] and 2P5B [24]) aligned with c-PHF8 by the Dali server [26].

4C). Therefore, the conformation of $\alpha 15, \beta 2$ and $\beta 3$ in c-PHF8, which are loops in JHDM1a, are not suitable for the binding of H3K36 (Figure 2B). Examination of the C-terminal sequences $(\alpha 14-\alpha 16)$ of PHF 8 and JHDM1a indicated that the $\mathrm{C}$-terminal sequence corresponds to a region of low sequence homology. The above differences may account for the substrate preference of these two demethylases. Second, the PHD domain of JHDM1a is located at the $\mathrm{C}$-terminal end, in an order opposite the arrangement in c-PHF8. The PHD domain of JHDM1a partially inhibits the enzymatic activity and is far away from the catalytic-core domain $[4,23]$. In contrast, the PHD domain of PHF8, which is adjacent to the JMJC domain, might enhance H3K9 demethylase activity through histone binding. Finally, the JMJC domain of c-PHF8 is stabilized by a hydrophobic core, composed of F246, I248, F279, W282, F292, F293, I318, I349 and L353 (Figure 3). The hydrophobic interaction in c-PHF8 is more extensive than in JHDM1a, which supports the structural stability of c-PHF8 in the absence of any other stabilizing structure, such as the additional C-terminal fragments in JHDM1a [23]. Taken together, these structural features distinguish the structure of c-PHF8 from that of the other two JMJC domain-containing proteins.

\section{Structural features that contribute to enzymatic specificities}

To understand why c-PHF 8 does not have activity on trimethyl H3K9, VOIDOO [27] was employed to calculate the volume of the active chamber, including the iron ion and $\alpha-K G$. The calculated cavity volume is $722.36 \AA$ [3] for c-JMJD2a [24, 25, 28], while they are 169.10 and $246.96 \AA$ [3] for c-PHF8 and JHDM1a, respectively. Therefore, the active chambers are too narrow for c-PHF 8 and JHDM1a to accommodate a trimethyl group. In c-PHF8, the large side chains of Y257 and F266 also make the cavity crowded. Moreover, the aromatic rings of F250 and Y234 narrow the substrate- binding channel, blocking entry of the trimethyl group into the active center (Figure 3 ).

To understand why PHF8 and JHDM1a have different substrate specificity, the structures of c-PHF 8 and JHDM1a were aligned with the complex structures of c-JMJD2a $[24,25,28]$ using the Dali server. Their surfaces are shown in Figure 4. The long and large side chains of residues K117, K323 and F324 blocked binding of the methyl H3K9 peptide to the JHDM1a surface (Figure 4B). This demonstrates that JHDM1a does not have activity on methyl H3K9 peptide due to the inability of binding. In a similar way, the conformation of $\alpha 15$ in c-PHF 8 might block the binding of H3K36 to PHF8 (Figure 4C, Supplementary information, Figure S3). In addition, hydrophilic interactions between the involved residues would play important roles in substrate recognition. For example, a positively charged residue Arg 117 in JHDM1a promotes the binding of negatively charged Tyr41 in H3K36. By contrast the corresponding residue is a negatively charged residue Asp167 in PHF8, and the repulsing force may inhibit the binding of H3K36 (Figures 2B and 4C). Taken together, these findings indicate that methylated $\mathrm{H} 3 \mathrm{~K} 36$ is not a good substrate for PHF8.

In our effort to dock an $\mathrm{H} 3 \mathrm{~K} 9 \mathrm{me} 2$ peptide aligned from the JMJD2A crystal (PDB:2OX0) onto the c-PHF8 structure, it appears that their interactions are not optimal (Figure 4C). PHD domains have been shown to bind histone tails $[21,22]$. The mouse PHF8 PHD structure (PDB:1WEP) is most closely related to that of BPTF [22], which has been shown to bind to trimethyl H3K4. Since $\mathrm{H} 3 \mathrm{~K} 4$ is spatially close to $\mathrm{H} 3 \mathrm{~K}$, a possible explanation may be that the PHD domain of PHF 8 will recognize H3K4me3, thus enhancing substrate binding. Therefore, it is possible that PHF8 is recruited to chromatin by the PHD domain and then alters specific histone modifications. 
Functional analysis of PHF8 mutant in disease

Recently, truncating mutations in human PHF8 were identified in patients $[11,13]$. An early stop codon induced by a 12 base-pair (bp) deletion at the exon $8 /$ intron 8 junction [11] and nonsense mutations R211X and K177X [13] were found in male patients with MR associated with cleft lip/palate. These nonsense mutations resulted in the loss of the entire JMJC domain in PHF 8 protein and therefore abrogate enzymatic activity. Generally, H3K9 methylation is associated with transcriptional repression, indicating that PHF8 may function to antagonize H3K9 methylation, which is associated with gene activity. In addition, deletion of the PHF8 ortholog 4F429 in Caenorhabditis elegans causes embryonic lethality [10]. Thus, these above findings suggest an important developmental role for $P H F 8$.

Interestingly, one exception is the F279S point mutation [29]. The clinical phenotypes of F279S patients were characterized by mild MR, mild dysmorphic features, unilateral cleft lip and cleft palate in one and bilateral cleft lip and cleft palate in the other sibling. The cause of these phenotypes remained to be identified. From the crystal structure, F279 is in the hydrophobic network surrounded by F246, I248 and I318, located on the loop forming the active center (Figure 3). We suspect that a change of hydrophobic F279 to hydrophilic serine would alter the above hydrophobic network and impact the active center structure, thus, inactivating the enzyme. To test the role of F279, the F279S mutant was expressed. The F279S mutant completely lost enzymatic activity (Figure 1A) compared to wild-type PHF8. Therefore, the enzymatic activity of PHF 8 likely plays an important role in cognitive function, midline formation and mental development.

In summary, we have shown that PHF 8 is a novel H3K9me2/1 demethylase. The structure of the catalyticcore domain of PHF8 reveals a conserved overall fold of the JMJC domain-containing demethylases, and it uses a distinctive hydrophobic core to stabilize the structure. The PHD domain is likely involved in binding the substrate to facilitate efficient demethylation. An examination of the structure shows that the diseasecausing mutation of PHF 8 causes an alteration to the active center of the enzyme. Thus, our structural and biochemical results presented here have provided mechanistic insights into the molecular function of PHF8 and the pathogenesis of PHF8 aberration in humans.

\section{Materials and Methods}

\section{Protein expression, purification and crystallization}

DNA fragments encoding various amino acid forms of wild- type human PHF8 or its mutants were amplified by PCR and ligated into pGEX-4T-2(GE Healthcare) and pet-28b (Novagen). The final clones were verified by restriction enzyme digestion and DNA sequencing. The recombinant plasmid containing the $c$ PHF8 gene was transformed in Rosetta 2 (DE3) cells (Novagen). Expression was induced by addition of IPTG (isopropyl-1-thiob-D-galactopyranoside) to an $8-1$ growing culture at $20^{\circ} \mathrm{C}$ when the $\mathrm{OD}_{600}$ reached 0.8 . After $8 \mathrm{~h}$ of additional growth, cells were harvested and resuspended in buffer A (20 mM Tris- $\mathrm{HCl}(\mathrm{pH} 7.2)$, $300 \mathrm{mM} \mathrm{NaCl}$ ) supplemented with PMSF (Invitrogen). After cell lysis by sonication and centrifugation, soluble pet-28b PHF8 was purified with His affinity beads (GE Healthcare). To produce a stable fragment suitable for crystallization, the purified protein was treated with trypsin $(0.1 \mathrm{mg} / \mathrm{ml})$ overnight at $4{ }^{\circ} \mathrm{C}$. N-terminal sequencing (Procise 491, ABI) and mass spectrometry (autoflex II TOF/TOF, Bruker Daltonics) identified it as residues 77-447 of the PHF8 protein. The PHF8 sample was loaded onto a Mono Q column (Pharmacia). After elution with a $\mathrm{NaCl}$ gradient, the homogeneity of the protein was examined using Coomassie blue-stained SDS-polyacrylamide gels. PHF8 was further purified with a Superdex 200 column (GE Healthcare). Fractions containing the protein were pooled and concentrated to $8 \mathrm{mg} / \mathrm{ml}$. c-PHF $8(8 \mathrm{mg} / \mathrm{ml})$ was crystallized by vapor diffusion against $200 \mathrm{mM} \mathrm{KCl}, 100 \mathrm{mM}$ Mes (pH 6.5) and 20\% PEG5K at room temperature. Crystals of the complex were prepared by soaking or co-crystallizing c-PHF8 in the above solution with $5 \mathrm{mM} \mathrm{H} 3 \mathrm{~K} 9 \mathrm{me} 2,1.7 \mathrm{mM} \alpha-\mathrm{KG}$ and $1 \mathrm{mM} \mathrm{Fe}^{2+}$, overnight.

For data collection, crystals were gradually transferred to a cryobuffer (reservoir buffer supplemented with $20 \%$ glycerol) and flash frozen in liquid $\mathrm{N}_{2}$. All data used in structure solution and refinement were collected at beamline BL17U1 at the Shanghai Synchrotron Radiation Facility or NE3A in the Photon Factory. Data were integrated and scaled with the HKL2000 suite of programs [30]. Data collection statistics are shown in Supplementary information, Table S1.

\section{Structure determination and refinement}

Using the structure of JHDM1a as the search model (PDB 2YU2) [23], molecular replacement solutions were found using PHASER [31] and MOLREP [32]. The crystal contains one protein per asymmetric unit, giving a crystal solvent content of $54 \%$. The model was built manually in the program COOT [33], and refinement was carried out with CNS [34] and REFMAC5 [35]. The final model contains amino acid residues 91-445 of PHF8 and the R factor is improved by TLSMD analysis [36]. Structure refinement statistics are shown in Supplementary information, Table S1. The structure of c-PHF8 with $\alpha-\mathrm{KG}$ was determined by the difference Fourier method and was refined to a resolution of $2.1 \AA$ (Supplementary information, Table S1).

\section{Demethylation activity assays}

Bulk histones $(2 \mu \mathrm{g})$ or $10 \mu \mathrm{M}$ of synthetic dimethyl histone peptide H3K9 (ARTKQTARK STGGKAPRKQLATKAARGGKbiotin) were incubated with purified GST-tagged PHF8 $(20 \mu \mathrm{g})$ in $50 \mu 1$ demethylation buffer $(20 \mathrm{mM}$ Tris- $\mathrm{HCl}(\mathrm{pH} 7.3), 150$ $\mathrm{mM} \mathrm{NaCl}, 50 \mu \mathrm{M}\left(\mathrm{NH}_{4}\right)_{2} \mathrm{Fe}\left(\mathrm{SO}_{4}\right)_{2}, 1 \mathrm{mM}$ DTT, $1 \mathrm{mM} \alpha-\mathrm{KG}, 2$ $\mathrm{mM}$ ascorbic acid) at room temperature. Reaction mixtures were analyzed by western blotting, MALDI-TOF or formaldehyde release assays (see Supplementary information, Data S1). The co- 
ordinates have been deposited in the PDB databank as $3 \mathrm{~K} 3 \mathrm{~N}$ and $3 \mathrm{~K} 3 \mathrm{O}$.

\section{Acknowledgments}

We thank Dr Dawei Li (China Agricultural University) for generously providing us with the experimental conditions during the early stages of this project. We thank Dr Ruiming Xu (Institute of Biophysics, Chinese Academy of Sciences) for critical reading of this manuscript and advice. We thank Dr Pinchao Mei (Chinese Academy of Medical Sciences and Peking Union Medical College), Xinqi Liu (Nankai University) and Jiemin Wong (East China Normal University) for discussions and advice. The synchrotronradiation experiments were performed at Shanghai Synchrotron Radiation Facility (SSRF) and NE3A in the Photon Factory. Z.C. is supported by the National Basic Research Program of China (973 Program, 2009CB825501), the National Natural Science Foundation of China (30870494 and 90919043), the New Century Excellent Talents in University (NCET-07-0808) and the Innovative Project of SKLAB. S. H. is supported by the National Key Laboratory Special Fund 2060204. Z. D. is supported by the National Natural Science Foundation of China (J0730639).

\section{References}

1 Zhang Y, Reinberg D. Transcription regulation by histone methylation: interplay between different covalent modifications of the core histone tails. Genes Dev 2001; 15:2343-2360.

2 Klose RJ, Kallin EM, Zhang Y. JmjC-domain-containing proteins and histone demethylation. Nat Rev Genet 2006; 7:715727.

3 Shi Y, Lan F, Matson C, et al. Histone demethylation mediated by the nuclear amine oxidase homolog LSD1. Cell 2004; 119:941-953.

4 Tsukada Y-i, Fang J, Erdjument-Bromage H, et al. Histone demethylation by a family of JmjC domain-containing proteins. Nature 2006; 439:811-816.

5 Whetstine JR, Nottke A, Lan F, et al. Reversal of histone lysine trimethylation by the JMJD2 family of histone demethylases. Cell 2006; 125:467-481.

6 Yamane K, Toumazou C, Tsukada Y-i, et al. JHDM2A, a JmjC-containing H3K9 demethylase, facilitates transcription activation by androgen receptor. Cell 2006; 125:483-495.

7 Xiang Y, Zhu Z, Han G, et al. JMJD3 is a histone H3K27 demethylase. Cell Res 2007; 17:850-857.

8 Schneider R, Bannister AJ, Kouzarides T. Unsafe SETs: histone lysine methyltransferases and cancer. Trends Biochem Sci 2002; 27:396-402.

9 Liesbeth ES, Ben CJH, Hans van B, et al. X-linked mental retardation associated with cleft lip/palate maps to Xp11.3q21.3. Am J Med Genet 1999; 85:216-220.

10 Cloos PAC, Christensen J, Agger K, Helin K. Erasing the methyl mark: histone demethylases at the center of cellular differentiation and disease. Genes Dev 2008; 22:1115-1140.

11 Laumonnier F, Holbert S, Ronce N, et al. Mutations in PHF8 are associated with $\mathrm{X}$ linked mental retardation and cleft lip/ cleft palate. J Med Genet 2005; 42:780-786.

12 Qiao Y, Liu X, Harvard C, et al. Autism-associated familial microdeletion of Xp11.22. Clin Genet 2008; 74:134-144.
13 Abidi FE, Miano MG, Murray JC, Schwartz CE. A novel mutation in the $P H F 8$ gene is associated with X-linked mental retardation with cleft lip/cleft palate. Clin Genet 2007; 72:1922.

14 Fernandez AG, Gunsalus KC, Huang J, et al. New genes with roles in the $C$. elegans embryo revealed using RNAi of ovaryenriched ORFeome clones. Genome Res 2005; 15:250-259.

15 Allis CD, Berger SL, Cote J, et al. New nomenclature for chromatin-modifying enzymes. Cell 2007; 131:633-636.

16 Elkins JM, Hewitson KS, McNeill LA, et al. Structure of factor-inhibiting hypoxia-inducible factor (HIF) reveals mechanism of oxidative modification of HIF-1 $\alpha$. J Biol Chem 2003; 278:1802-1806.

17 Lee C, Kim SJ, Jeong DG, Lee SM, Ryu SE. Structure of human FIH-1 reveals a unique active site pocket and interaction sites for HIF-1 and von Hippel-Lindau. J Biol Chem 2003; 278:7558-7563.

18 Chen Z, Zang J, Whetstine J, et al. Structural insights into histone demethylation by JMJD2 family members. Cell 2006; 125:691-702.

19 Klose RJ, Yamane K, Bae Y, et al. The transcriptional repressor JHDM3A demethylates trimethyl histone H3 lysine 9 and lysine 36. Nature 2006; 442:312-316.

20 Cloos PAC, Christensen J, Agger K, et al. The putative oncogene GASC1 demethylates tri- and dimethylated lysine 9 on histone H3. Nature 2006; 442:307-311.

21 Lan F, Collins RE, De Cegli R, et al. Recognition of unmethylated histone $\mathrm{H} 3$ lysine 4 links BHC80 to LSD1-mediated gene repression. Nature 2007; 448:718-722.

22 Li H, Ilin S, Wang W, et al. Molecular basis for site-specific read-out of histone $\mathrm{H} 3 \mathrm{~K} 4 \mathrm{me} 3$ by the BPTF PHD finger of NURF. Nature 2006; 442:91-95.

23 Han Z, Liu P, Gu L, et al. Structural basis for histone demethylation by JHDM1. Frontier Sci 2007; 1:52-61.

24 Chen Z, Zang J, Kappler J, et al. Structural basis of the recognition of a methylated histone tail by JMJD2A. Proc Natl Acad Sci USA 2007; 104:10818-10823.

$25 \mathrm{Ng}$ SS, Kavanagh KL, McDonough MA, et al. Crystal structures of histone demethylase JMJD2A reveal basis for substrate specificity. Nature 2007; 448:87-91.

26 Holm L, Kaariainen S, Rosenstrom P, Schenkel A. Searching protein structure databases with DaliLite v.3. Bioinformatics 2008; 24:2780-2781

27 Kleywegt GJ, Jones TA. Detection, delineation, measurement and display of cavities in macromolecular structures. Acta Crystallogr D 1994; 50:178-185.

28 Couture JF, Collazo E, Ortiz-Tello PA, Brunzelle JS, Trievel RC. Specificity and mechanism of JMJD2A, a trimethyllysinespecific histone demethylase. Nat Struct Mol Biol 2007; 14:689-695.

29 Koivisto AM, Ala-Mello S, Lemmelä S, et al. Screening of mutations in the $P H F 8$ gene and identification of a novel mutation in a Finnish family with XLMR and cleft lip/cleft palate. Clin Genet 2007; 72:145-149.

30 Otwinowski Z, Minor W. Processing of X-ray diffraction data collected in oscillation mode. Methods Enzymol 1997:307-326.

31 McCoy AJ, Grosse-Kunstleve RW, Adams PD, et al. Phaser crystallographic software. J Appl Crystallogr 2007; 40:658674. 
32 Vagin A, Teplyakov A. MOLREP: an automated program for molecular replacement. J Appl Crystallogr 1997; 30:10221025.

33 Emsley P, Cowtan K. Coot: model-building tools for molecular graphics. Acta Crystallogr D 2004; 60:2126-2132.

34 Brunger AT, Adams PD, Clore GM, et al. Crystallography \& NMR system: a new software suite for macromolecular structure determination. Acta Crystallogr D 1998; 54:905-921.
35 Murshudov GN, Vagin AA, Dodson EJ. Refinement of macromolecular structures by the maximum-likelihood method. Acta Crystallogr D 1997; 53:240-255.

36 Painter J, Merritt EA. Optimal description of a protein structure in terms of multiple groups undergoing TLS motion. Acta Crystallogr D 2006; 62:439-450.

37 Barton GJ. ALSCRIPT: a tool to format multiple sequence alignments. Protein Eng 1993; 6:37-40.

(Supplementary information is linked to the online version of the paper on the Cell Research website.) 